\title{
La teoría fundamentada en busca de un modelo educativo Qulla-Aymara
}

\author{
Nelson Cutipa Gómez ${ }^{1}$ \\ https://orcid.org/0000-0002-5818-5584
}

\section{Resumen}

El presente estudio se fundamenta a partir de la concepción del yati (sabe) del mundo qulla (aymara). El hallazgo de elementos de un proceso cognoscitivo propio de los qulla (aymaras) convoca a la sistematización de un nuevo paradigma educativo con bases filosóficas propias, con una epistemología emergida del pensamiento de los qulla (aymaras) a partir de su idioma aymara. La reestructuración de un modelo educativo intra e intercultural intraeducativo e intereducativo es de vital importancia en estos momentos de revolución educativa de nuestro Estado Plurinacional de Bolivia. El YATI conlleva distintos significados y significantes en el proceso aprendizaje. A través del discurso de los aymara hablantes de tres regiones (CariquimaChile, Tacna-Perú y Ingavi-Bolivia) se ha podido evidenciar un continuum de códigos y las redes del software de Atlas.ti ha podido sistematizar los códigos, las parte del YATI a través de redes semánticas en torno al yati 'sabe' los cuales son; amta-recuerdo, amuyu-pensamiento, lup'i-razonamiento y lura-hacer. El estudio ha podido aproximar a una secuencia lógica de eventos sincronizados para acceder a un proceso cognoscitivo a partir de su significancia propia del idioma Qulla-Aymara y un intento de poder dar pautas de cómo debe entenderse el proceso yati 'sabe' en el mundo andino de la región de los Aymaras, como una propuesta de método del Proceso Aprendizaje Aymara PÄ.

Palavras Clave: Modelo, Aprendizaje, Enseñanza, Yati

\section{Grounded theory in search of a Qulla-Aymara educational model}

\section{Abstract}

The present study is based on the conception of the yati (knows) of the world qulla (aymara). The discovery of elements of a cognitive process typical of the Qulla (Aymaras) calls for the systematization of a new educational paradigm with its own philosophical bases, with an epistemology emerged from the thinking of the Qulla (Aymaras) from their Aymara language. The restructuring of an intra and intercultural intra-educational and inter-educational model of education is of vital importance in these moments of educational revolution in our Plurinational State of Bolivia. The YATI carries different meanings and signifiers in the

\footnotetext{
${ }^{1}$ Docente Investigador (Carrera de Lingüística e Idiomas) da Universidad Mayor de San Andrés - UMSA (La Paz - Bolivia). Estudiante del Programa Doctorado no Escolarizado en Educación Superior del Centro Psicopedagógico y de Investigación en Educación Superior (CEPIES/UMSA). Licenciado en Lingüística e idiomas. E-mail: nelsoncutipagomezumsa@hotmail.com.
} 
learning process. Through the speech of the Aymara speakers from three regions (CariquimaChile, Tacna-Peru and Ingavi-Bolivia) a continuum of codes has been evidenced and the software networks of Atlas.ti have been able to systematize the codes, the parts of the YATI through semantic networks around the yati 'knows' what they are; amta-memory, amuyuthought, lup'i-reasoning and lura-do. The study has been able to approximate a logical sequence of synchronized events to access a cognitive process based on its significance in the Qulla-Aymara language and an attempt to provide guidelines for how the yati 'knows' process should be understood in the Andean world. from the Aymara region, as a proposed method of the Aymara PÄ Learning Process.

Keywords: Model, Learning, Teaching, Yati.

\section{Los modelos educativos emergieron a partir de las necesidades humanas en diferentes perspectivas}

Un modelo educativo actúa sobre el desarrollo del pensamiento humano frente a la realidad que le circunda. Cada modelo educativo está presente en la humanidad a partir de reflexiones y necesidades de cada pueblo, nación o Estado. Así también cada modelo educativo según a la disciplina desarrolla nuevos significados y significantes, conceptos especializados para manipular a su objeto u sujeto, a través de métodos, en busca de nuevas alternativas educativas.

Cuando nos referimos a modelos educativos nos referimos a modelos de pensamiento, sistemas de cognición que se han desarrollado en beneficio de la humanidad. Los modelos de aprendizaje o enseñanza son temas técnicos, al igual como la didáctica establece estrategias para cumplir y alcanzar ese modelo de pensamiento.

Edgar Morin entiende al sujeto (ser humano) como complejo y multidimensional, simultáneamente homo sapiens, faber, demens, ludens y mythologicus. Para Suanno (Suanno, 2015, pág. 81) dicha perspectiva "amplia el concepto de ser humano y lo comprende como un ser histórico, social, cultural, pero también biológico, cognitivo, pleno de subjetividad, psicoafectivo y enigmático, ser de razón, emoción y corporeidad”.

Desde luego el aprendizaje es un proceso social, cultural, histórico, fisiológico y psicológico que se encuentra al interior del ser. Mientras que el modelo educativo está diseńado para que pueda desarrollar el aprendizaje o enseñanza bajo un modelo diseñado una sociedad, es decir, cómo dejar un modelo de vida para su continuidad y desarrollo de convivencia humana en el mundo. (Morin, 2011) 
Los modelos de educación a lo largo de la historia de la humanidad indagaron actitudes, aptitudes, destrezas, en fin, necesidades cognitivas para alcanzar el conocimiento y dar continuidad y el desarrollo del saber sobre lo desconocido.

La enseñanza del experto y el aprendizaje del novato debieron facilitar el aprendizaje, pero en la actualidad aún la enseñanza sigue siendo el problema de la educación. Mientras el aprendizaje solo fue estudiado a partir de la enseñanza y no a partir del aprendizaje, desde el sujeto que tiene la verdadera responsabilidad del conocer no por obligación, mas al contrario por un deber, una obligación tras una decisión individual o grupal.

\section{El modelo de la enseńanza tradicional}

La memorización como una asociación o una mnemotecnia ${ }^{2}$. La narración, las preguntas como método de enseñanza fue para sistematizar lo conocido o lo que otros conocen y se debe repetir de esa forma bajo una forma de retahíla de personajes, fechas y sucesos. La Mayéutica como método de enseñanza a través del diálogo entre el interpelado y él interlocutor. El tradicionalismo de las civilizaciones de Grecia y Romana permitieron repetir; cómo pasó, pasará, y repasará el conocimiento que se enseña y el interlocutor aprende. La ciencia exacta debe ser repetitiva, al igual que el positivismo reiterativo. De esta forma se mantiene la enseñanza bajo un modelo memorístico, que reitera todo en torno a un ideal o modelo de modelos memorísticos llamado marco teórico, o estado de arte, Theoretical framework. La universitas lat. 'universidad' precisamente universaliza el conocimiento bajo la memorización de las teorías euro centristas.

\section{El modelo de la enseñanza conductista}

La estimulación refleja una condición y como resultado tenemos una respuesta. El control, la programación del comportamiento y toda actividad humana estaba condicionada. Algunos basan esta enseńanza en un estímulo

\footnotetext{
${ }^{2}$ La palabra «Mnemotecnia» deriva del griego mnéemee (memoria), y téchnee (arte). Una regla mnemotécnica es un sistema sencillo utilizado para recordar una secuencia de datos, nombres, números, y en general para recordar listas de datos relacionando palabras, imágenes, etc.
} 
respuesta, basado en un ensayo y respuesta. Algunos agregaron el reforzamiento. Para Rusia, el fin es planificar y tener el control de una sociedad utópica, en busca de una sociedad perfecta y controlada a través de estímulos y refuerzos positivos. "Los fenómenos fisiológicos son reducidos a hechos fisiológicos”. (Cutipa, 2013, pág. 62)

La enseńanza conductista tiene como resultado la enseñanza programada, entornos virtuales como tecnología educativa y el desarrollo de Nuevas Tecnologías de la Información y la Comunicación (NTIC) como una propuesta del neoconductismo.

\section{El modelo de la enseñanza por descubrimiento}

La enseñanza ha fracasado y se busca una nueva enseñanza significativa, porque, todo lo que hizo hasta el momento es repetir lo que ya fue creado. La enseñanza de hoy debe ser crítica, que descubra, analice y critique. A partir de una realidad propia del individuo. La enseñanza solo es guiada donde la enseñanza es un proceso de construcción de modelos y reglas de presentación. La enseñanza y aprendizaje es activa a partir de sus acciones de asimilación.

La enseñanza encamina al alumno a que descubra por sí mismo su carácter individual en su aprendizaje a través de la enseñanza para que descubra por si mismo y despertar el carácter investigativo y exploratorio del alumno.

Asimismo, se ha criticado la enseñanza por descubrimiento por ser elitista, porque solo unos pocos podrán tener el conocimiento.

Cuanto más significativos sean nuestras actividades mayores será nuestro aprendizaje.

\section{El principio de la enseńanza limita el aprendizaje}

La enseńanza es un problema ajeno al ser, el problema real del ser es el aprendizaje. El ser humano al identificarse como una especie superior a las demás especies busca ser un ser particular único.

La teoría occidental euro centrista ha mostrado que la enseñanza es una pedagogía para la continuidad de su especie y su conocimiento. La universalización de esos preceptos llegó al Awiyala (América) por inercia y en la Colonia, República y hasta nuestros días. 
El conocimiento de las distintas disciplinas estaba en manos de terceros que enseñaron a ser como europeos y sin serlos nuestro proceso de cognición en las distintas disciplinas son desde el punto de vista europeo o norteamericano. No pudieron abordar, desarrollar y menos transformar el pensamiento, conocimiento, técnica, tecnología, ciencia propia a partir de nuestra percepción. Es decir, la ciencia indígena (indio) hasta nuestros días sigue en un statu quo. En la Republica nos enseñaron a ser hijos de Bolívar y luego nacionalistas y no lo lograron transformarnos. Hoy tenemos una educación intercultural plurilingüe y no saben a que cultura pertenecen y menos ya hablan su idioma, pero si entienden la lengua.

El problema de américa latina fue la enseñanza, y peor los resultados del aprendizaje unas peleas con el subconsciente del sin luz que vivía en la oscuridad de la caverna de Platón. Pero, la institución social y los resabios de significados y significantes en el idioma aymara han logrado mantener viva la esencia del Aprendizaje Qulla-Aymara y de esta forma pervive el lexema Yati.

\section{Métodos de la investigación}

Los métodos en la presente investigación se destacan según la etapa investigativa. El fundamento metodológico de la presente tesis doctoral se fundamente dentro el enfoque cualitativo. A partir de Anselm Strauss y Juliet Corbin conceptualizamos la investigación cualitativa como; un medio para construir teoría por medio de los análisis de datos cualitativos que desean construir teoría en su nivel sustantivo. Cada ser humano, grupo, comunidad, sociedades tienen una distinta forma de pensar sobre cada realidad y la diversidad de muchos factores hace pensar distinto a cada ser humano conforme a su cultura heredada por generaciones y expresada por las distintas lenguas o idiomas que trata de conceptualizar las distintas realidades de cada grupo humano. (Strauss\&Corbin, 2002)

La investigación sustantiva precisamente se adscribe a la investigación cualitativa. Para la presente investigación el objeto de la investigación desde un punto de vista formal se transforma en el subjeto es decir el objeto validado 
como sujeto (un objeto con vida que nos informa sobre su estado y ánimo) por lo cual se le ha denominado con el nombre de subjeto. ${ }^{3}$

"La investigación que intenta comprender el significado o naturaleza de la experiencia de personas con problemas tales como enfermedad crónica, adicción, divorcio y el acto de "destaparse" se presta a trabajo de campo para encontrar lo que la gente hace y piensa. Los métodos cualitativos pueden usarse para explorar áreas sustantivas sobre las cuales se conoce poco o mucho, pero se busca obtener un conocimiento nuevo (Stern, 1980)." Citado en (Strauss\&Corbin, 2002, pág. 27)

Básicamente, existen tres componentes principales en la investigación cualitativa. Primero, están los datos, que pueden provenir de fuentes diferentes, tales como entrevistas, observaciones, documentos, registros y películas. Segundo, están los procedimientos, que los investigadores pueden usar para interpretar y Tercero organizar los datos. (Strauss\&Corbin, 2002)

El método relevante de la presente investigación es la teoría fundamentada (grounded theory). Según esta teoría “... consiste en relaciones posibles entre "conceptos" (1,2 y 3) y "conjunto de conceptos". Por ejemplo, cuando revisamos las grabaciones del trabajo de campo, es común observar un encadenamiento de ideas, desarrollado por el hablante. Una ida sigue a otra según la propia lógica del hablante (o de un lugar determinado) lo que habrá que llegar a identificar y entender." (Arnold, 2010, pág. 58)

Se refieren a una teoría derivada de datos recopilados de manera sistemática y analizada por medio de un proceso de investigación.

\section{Universo, población y muestra}

El universo de la presente investigación ha tomado en cuanta a determinado a los hablantes del idioma Aymara de tres países; República del Perú, República de Chile y Estado Plurinacional de Bolivia

La población de la presente investigación ha tomado en cuenta las tres variedades de habla aymara en el continente.

\footnotetext{
${ }^{3}$ Subjeto. Concepto propuesto por el investigador, es un lexema compuesto de dos constituyentes la primera es objeto y la segunda es sujeto y la fusión morfológica nos da el resultado de un neolexema subjeto, los mismos son los nombres que da las dos tendencias de la investigación la primera cuantitativa (objeto) y la segunda cualitativa (Sujeto) y la realidad subjetiva objetivada para su comprobación será denominada subjeto en la presente investigación.
} 
(1) La variedad norteña se ha tomado a la mayoría de los informantes en el Departamento de La Paz - Bolivia.

(2) La variedad intermedia se ha tomado a dos cooperantes de Tacna.

(3) Variedad Sureño a cooperantes de Chile.

(1) La Primera Sección Viacha, de la Provincia Ingavi, del Departamento de La Paz está situada a 32 kilómetros al suroeste de sede de gobierno con una vía de acceso por la carretera asfaltada La Paz - Viacha. Su geografía presenta un relieve ondulado, con presencia de serranías, con un clima favorable y temperaturas que oscilan entre $8^{\circ}$ a $10^{\circ} \mathrm{C}$. Con una altitud 3.853 m.s.m. y con precipitaciones fluviales de $500 \mathrm{~mm}$. Sus principales ríos son el desaguadero, Katar Jawira, Ch'ama, Jach'a Jawira y el río Pallina. (Limachi, 2011) La Provincia Ingavi cuenta con las siguientes Secciones: Primera -Viacha, Segunda-Guaqui, la Tercera -Tiahuanaco, Cuarta Desaguadero, Quinta - San Andrés de Machaca, Sexta- Jesús de Machaca y por último como séptimo - Taraco. La Primera Sección cuenta con seis Cantones y son; Viacha, Villa Remedios, Villa Santiago de Chacoma, Irpuma Irpa Grande, Hichuraya grande, Gral. José Ballivián. La capital de la Provincia Ingavi es Viacha. La Primera Sección cuenta con sesenta y tres comunidades. Según los archivos de la parroquia de Viacha antiguamente nuestro territorio estaba conformado por sayas Haransaya y Hurinsaya, estas eran subdivididas en ayllus y en la actualidad son comunidades. (Limachi, 2011)

(2) La región de Tacna, se encuentra aproximadamente a $400 \mathrm{Km}$ de la ciudad de La Paz - Bolivia, es parte de los 24 departamentos de la República del Perú. Se encuentra al sur de la capital del Perú. Conforme a la relación histórica aún mantiene su cultura tradicional aymara, ama teniendo su identidad pese a la resistencia a la chilenización. El significado del nombre de Takna en aymara significa jach'a suyu.

(3) La República de Chile, ubicada en la I Región de Tarapacá, el cual tiene dos provincias la Provincia de Iquique con su capital Iquique y la Provincia de Tamarugal con su capital Pozo Almonte. La comuna de Colchane tiene distritos como Isluga, Cariquima, Cariquima, Puchuldiza y Caico. El distrito Cariquima tiene aproximadamente 480 habitantes según el censo de 2002. Su característica es por tener el nevado Cariquima o Mama Huanapa. (Social, 2014) 
Tabla 1 Fuente: Adaptación. Reporte Comunal Colchane. Serie Informes Comunales $\mathrm{N}^{\circ} 1,7$ de Febrero 2014

\begin{tabular}{|l|l|l|}
\hline \multicolumn{1}{|c|}{ VARIEDAD NORTEÑO } & \multicolumn{1}{|c|}{$\begin{array}{c}\text { VARIEDAD } \\
\text { INTERMEDIO }\end{array}$} & \multicolumn{1}{|c|}{ VARIEDAD SUREÑO } \\
\hline Bolivia, La Paz & Perú, Tacna y Moquegua. & $\begin{array}{l}\text { Bolivia, Oruro, Potosí, } \\
\text { Cochabamba I }\end{array}$ \\
\hline Perú, Puno & $\begin{array}{l}\text { Chile, I Región Tarapacá, } \\
\text { Provincia Pozo Almonte, Comuna } \\
\text { de Colchane. }\end{array}$ \\
\hline $\begin{array}{l}\text { Chile, Comuna General } \\
\text { Lagos, Provincia Parinaquta, } \\
\text { XV Región. }\end{array}$ & & \\
\hline
\end{tabular}

La muestra de la presente investigación ha tomado los siguientes criterios:

Tener por lengua materna el aymara

- Ser parte de una comunidad, o comuna de la región aymara

- Tener manejo del idioma en sus actividades laborales, familiares

- Tener hijos o hijas

Los informantes de Bolivia fueron Qulla-Aymara(s) voluntarias de tipo nucleares, es decir familias donde conviven la madre, el padre y los hijos. La muestra se dividirá en sujetos cooperantes como ser los padres y madres.

Los informantes de Chile fueron féminas hablantes aymaras de la región de Cariquima. Todas las señoras actualmente son miembros del ANLA ${ }^{4}$.

Los informantes de Perú son mujeres y varones que se brindaron a ser los cooperantes para la investigación doctoral. El número de la muestra del Perú está establecido que todos sean docentes de la Universidad.

\section{Resultados}

A partir de las grabaciones transliteradas en un medio físico de análisis se procedió al vaciado de datos primarios. Los datos primarios en primera instancia fueron doce entrevistas, de cuales, solo un número de nueve

\footnotetext{
${ }^{4}$ Academia Nacional de la Lengua Aymara. Aymar Aru Sarayiri Sargento Aldea N 791 2do Piso. Iquique-Chile.
} 
cooperantes fueron los elegidos. ${ }^{5}$ Uno de los nueve se caracteriza por ser un audio en el cual se establece un análisis cualitativo de audio.

(1) El Yati un modelo educativo andino intercultural e intracultural codigos - ATLAS.ti

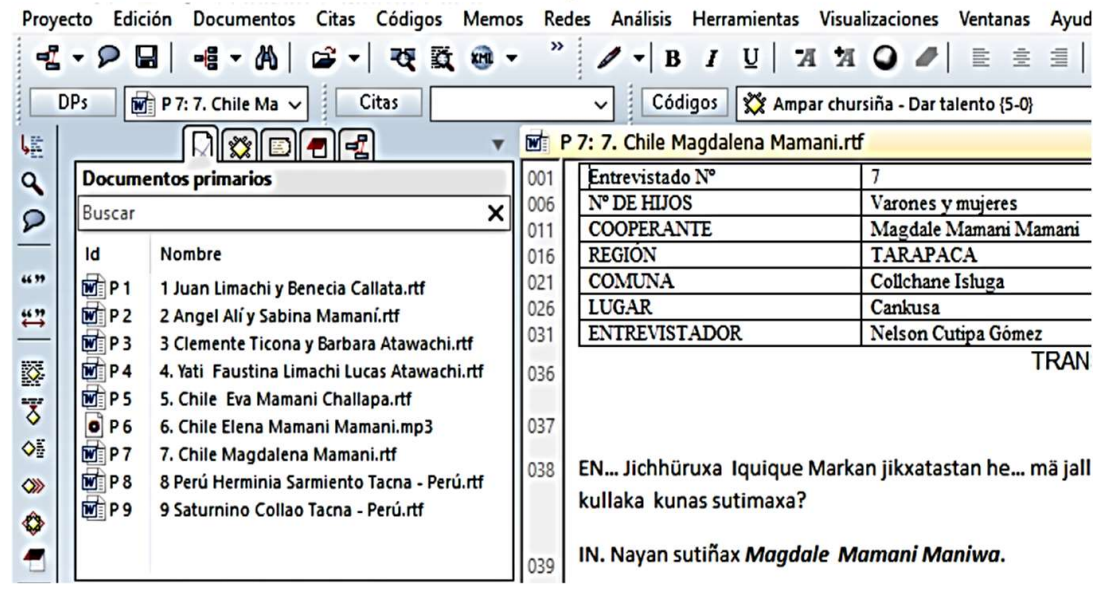

Los códigos fueron extraídos secuencialmente a partir de la oralidad y fueron cotejados por la frecuencia de uso en el lenguaje para describir la educción a partir del qulla-aymara.

Los códigos fueron sistematizados a partir de la acción establecidos en la categoría de verbos aymaras, los nombres o sustantivos que fueron empleados en el discurso aymara por los cooperantes.

\footnotetext{
${ }^{5}$ Se seleccionó las grabaciones legibles, es decir que tengan buen audio y tres de ellas tiene interferencias en el audio como ser el viento en el momento de la grabación ya que se lo realizó en campo abierto
} 
(2) El Yati un modelo educativo andino intercultural e intracultural codigos - ATLAS.ti

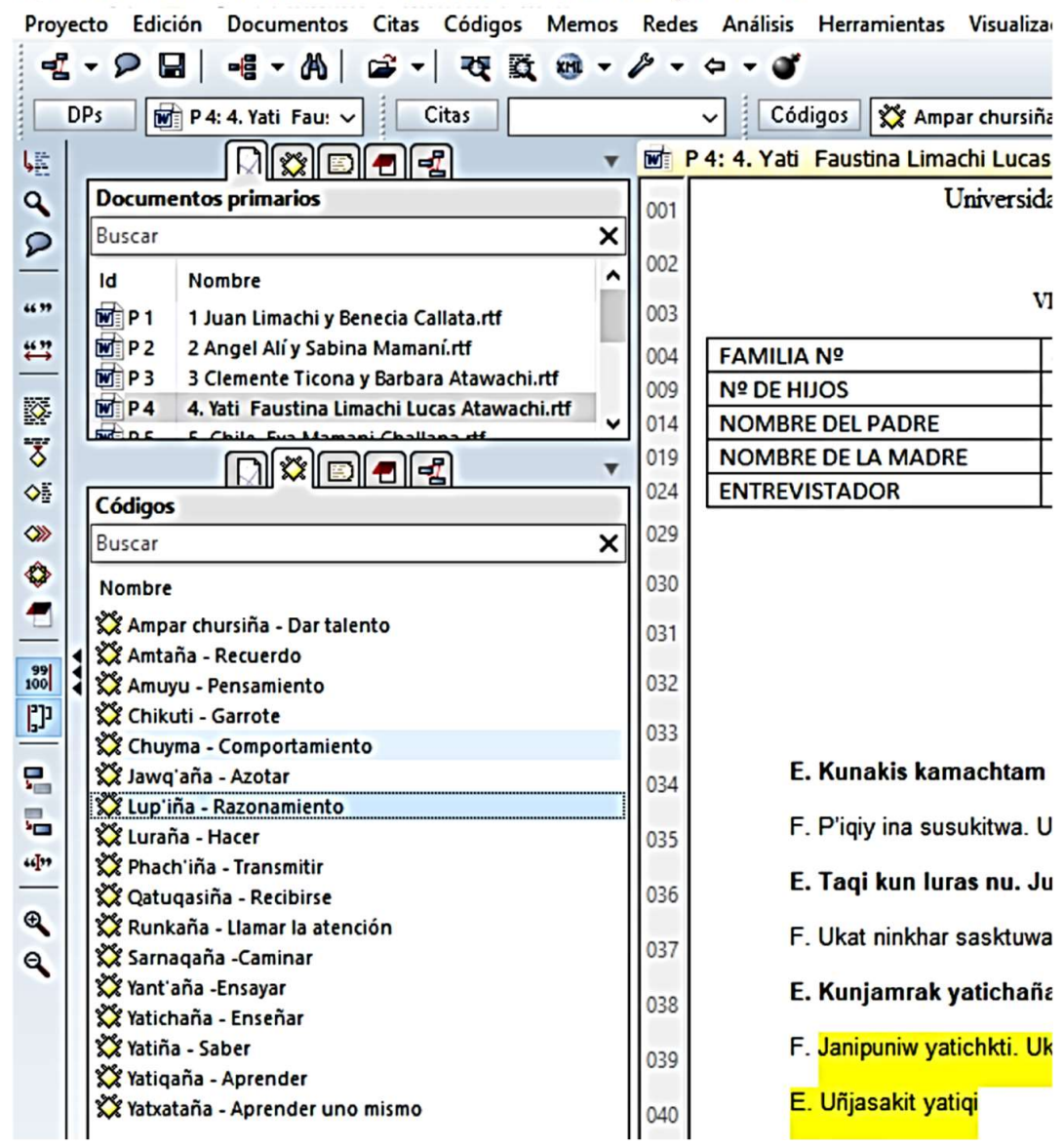

Los códigos generados fueron diez y siete, pero dos de los códigos son una actividad (Chikuti - jawq'aña).

Luego se procedió a categorizar cada una de las citas seleccionadas conforme a cada categoría que estaba relacionada con el proceso de adquisición de conocimientos. 


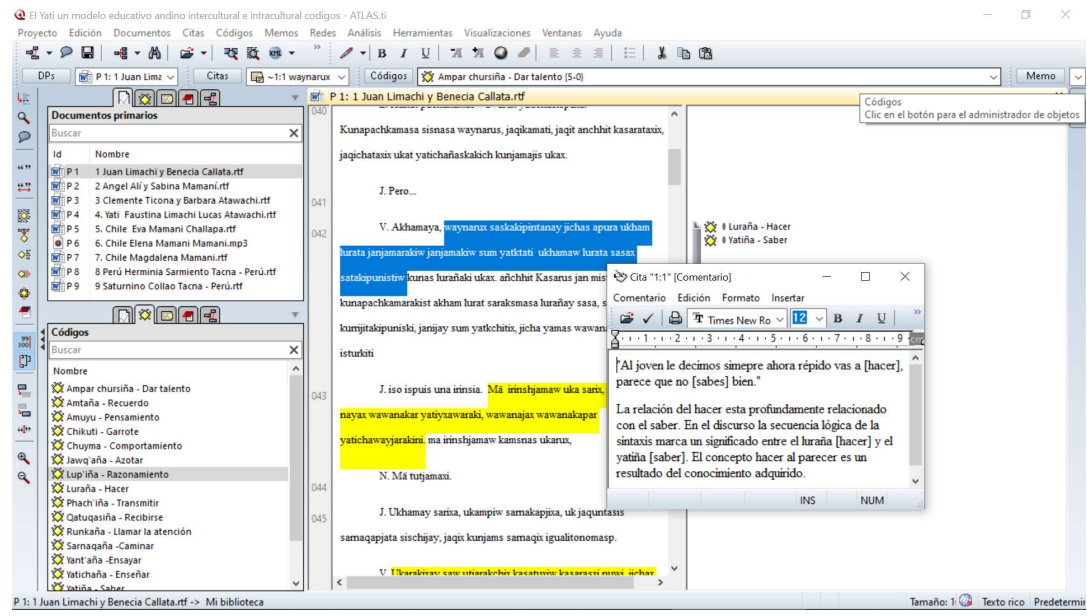

Dada línea esta enumerada y seleccionada por el investigador conforme a las pesquisas del objetivo de la presente investigación del proceso y la hermenéutica de cómo se adquiere el conocimiento en el mundo QullaAymara de los 9 informantes de distintos países miembros de una nación y hablantes de un idioma Aymara.

Las citas fueron seleccionadas a partir de los códigos y viceversa. La cita fue seleccionada de las reiteraciones de enunciados importantísimos que develan el proceso de adquisición de conocimientos.

Los comentarios fueron un espacio para poder realizar una traducción al idioma castellano para su comprensión y sistematización de los códigos mas impactantes que aproximan al descubrimiento del método de adquisición cognoscitiva para los Qulla-Aymaras.

En algunos se utilizó memos una especie de anotaciones más importantes y frágiles que puede perderse de la memoria del investigador y necesarias en el momento de la lectura de los hechos lingüísticos de cada cooperante ante la cuestionante de cómo se aprende o enseña al nuevo ser de la sociedad Qulla- Aymara. 
Administrador de citas [UH: backup of backup of El Yati un modelo educativo andino intercultural e intr. Citas Edición Miscelánea Imprimir Visualizar

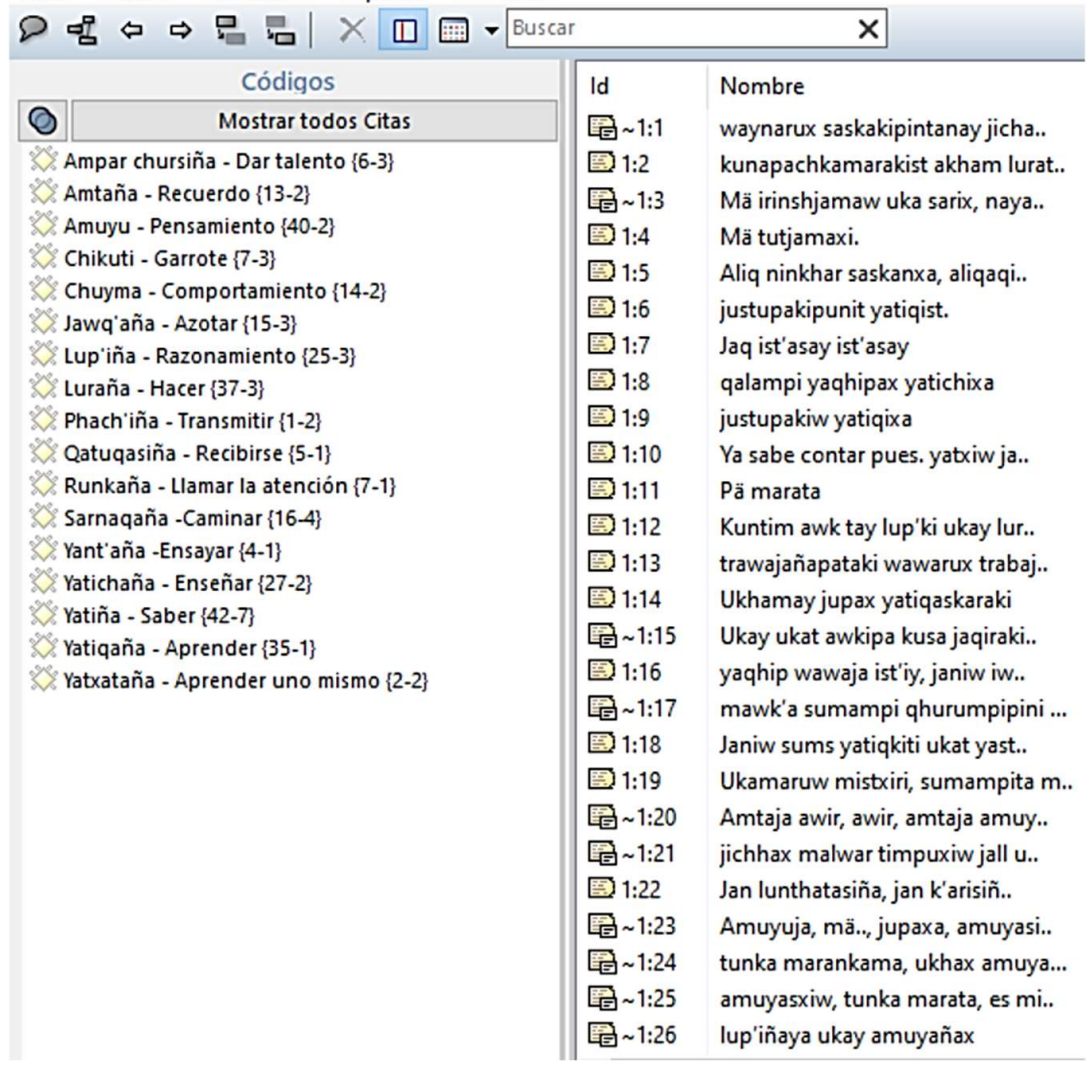

El administrador de citas nos muestra la recurrencia en yatińa-saber (41); amuyu-pensar (39) Luraña-hacer (36) yatiqaña-aprender (32); yatichaña-enseñar (30), lup'i-razonamiento (21); sarnaqaña-Caminar (13) y Amtaña-recuerdo (12).

A partir de las citas con referencia a los procesos cognoscitivos Qullaaymara se tiene una intención de conceptos más usuales en el idioma aymara para referirse al proceso de transferencia de conocimientos. 
El siguiente análisis fue por examinador de palabras, estas pueden analizar la frecuencia de uso de todos los nueve cooperantes. Por nube de frecuencias o por frecuencias sistematizadas en tablas de Excel.

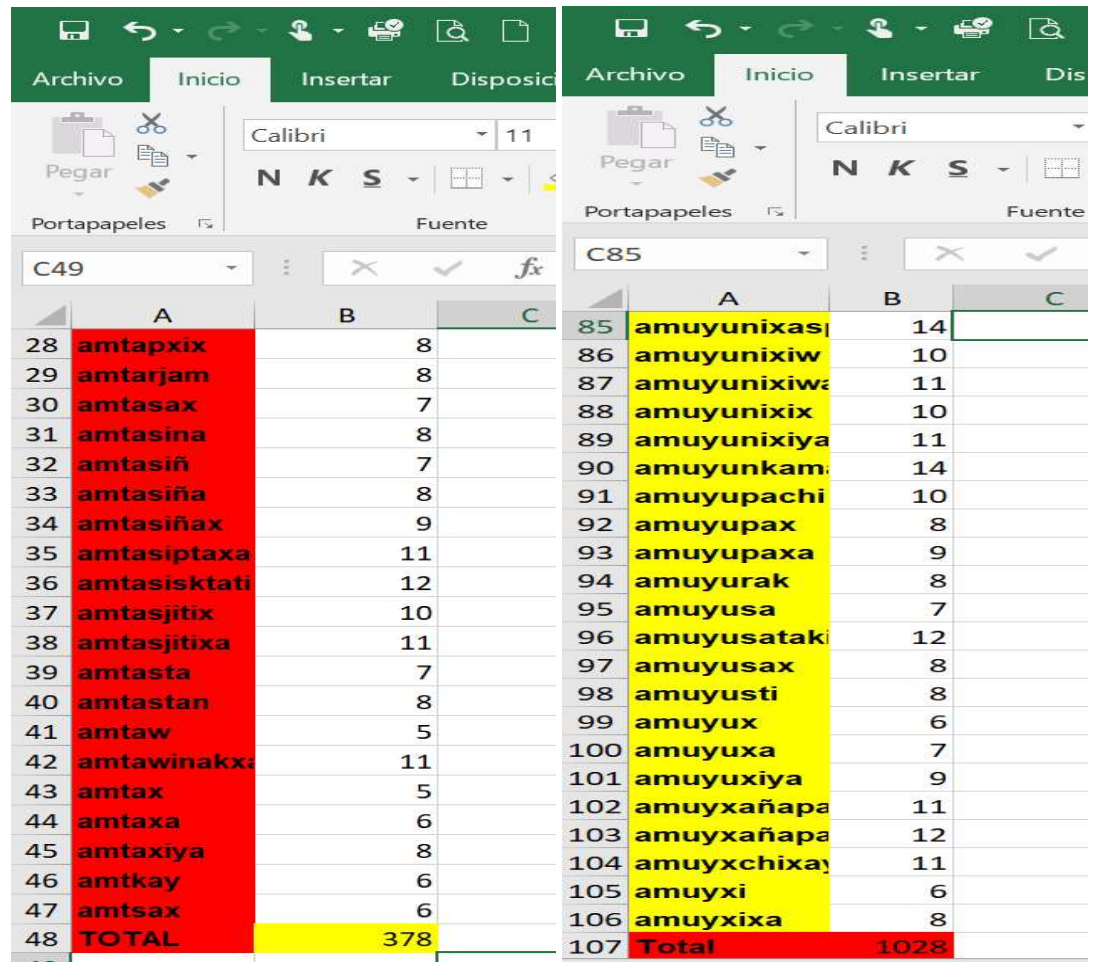

El idioma aymara tipológicamente al ser sufijante, aglutinante encapsulante muestra una serie de conjugaciones pero que esencialmente el tronco, raíz, el morfema base, el morfema libre es la esencia del significado yatiña-saber; amuyu-pensar; Luraña-hacer; yatiqaña-aprender; yatichañaenseñar, lup'i-razonamiento; sarnaqaña-Caminar y Amtaña-recuerdo.

La sistematización de la frecuencia de palabras fue de gran ayuda para poder aproximarnos a la recurrencia de los términos que más se emplea para poder explicar a través de la oralidad sobre el proceso cognoscitivo Qullaaymara. 
Las redes semánticas que el Microsoft del Atlas.ti a partir de los datos introducidos por el investigador fue importantísimo para poder develar el discurso en torno al proceso cognitivo qulla- aymara.

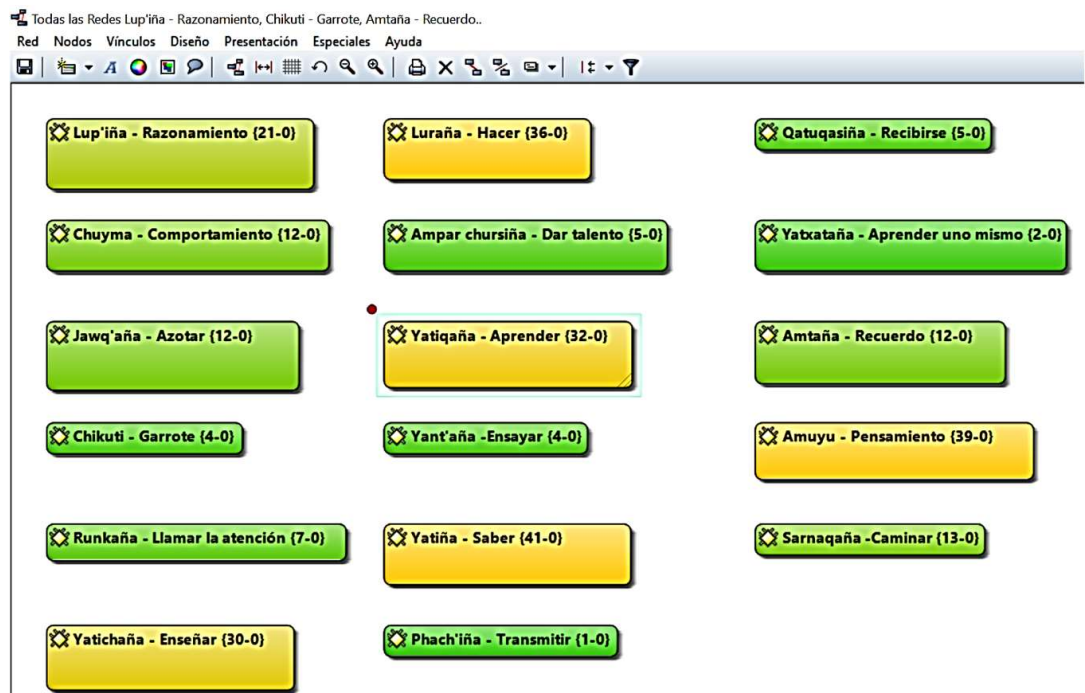

Las redes semánticas que posee mayor fundamento son seis, entre ellos está yatiqaña, yatiña, yatichaña, luraña, lup'iña, amuyu, amtaña, chuyma y jawq'aña.

\begin{tabular}{|c|c|c|c|}
\hline \multicolumn{4}{|c|}{$\begin{array}{l}\text { 23 Administrador de códigos [UH: El Yati un modelo educa } \\
\text { Códigos Edición Miscelánea Imprimir Visualizar }\end{array}$} \\
\hline 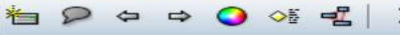 & $\times$ 且 & 口四 & -1 \\
\hline Nombre & \multicolumn{3}{|c|}{ Fundamentado } \\
\hline 25. Ampar chursiña - Dar talento & \multicolumn{3}{|l|}{ 르 } \\
\hline 18. Amtaña-Recuerdo & \multicolumn{3}{|l|}{$5=$} \\
\hline \$2 Amuyu - Pensamiento & \multicolumn{3}{|c|}{39} \\
\hline 3. Chikuti - Garrote & \multicolumn{3}{|l|}{ in } \\
\hline \$2. Chuyma - Comportamiento & \multicolumn{2}{|l|}{ 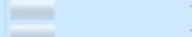 } & 12 \\
\hline 20. Jawq aña - Azotar & & 12 \\
\hline 30. Lup"iña - Razonamiento & \multicolumn{2}{|l|}{ sesen } & 21 \\
\hline 2. Luraña-Hacer & \multicolumn{3}{|l|}{ 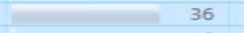 } \\
\hline $\begin{array}{l}\text { 28 Phach'iña - Transmitir } \\
\text { 28 Qatuqasiña - Recibirse }\end{array}$ & & 1 \\
\hline $\begin{array}{l}\text { 28 Qatuqasiña - Recibirse } \\
\text { 28. Runkaña - Llamar la atención }\end{array}$ & \multicolumn{2}{|l|}{ In } & 5 \\
\hline $\begin{array}{l}\text { 28. Runkaña - Llamar la atención } \\
\text { 25 Sarnagaña - Caminar }\end{array}$ & \multirow{2}{*}{\multicolumn{2}{|c|}{ Eing }} & 7 \\
\hline 2.5 Sarnaqaña -Caminar & & & 13 \\
\hline 28 Yant'aña -Ensayar & \multicolumn{2}{|l|}{ in } & 4 \\
\hline \$ี Vatichaña - Enseñar & \multicolumn{2}{|c|}{ 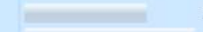 } & 30 \\
\hline 28: Yatiña - Saber & \multicolumn{3}{|c|}{41} \\
\hline \multirow{2}{*}{$\begin{array}{l}\text { 32. Vatiqaña - Aprender } \\
\text { \$2 Yatxataña - Aprender uno mismo }\end{array}$} & \multicolumn{3}{|c|}{ 践 } \\
\hline & & & 2 \\
\hline
\end{tabular}


A partir del número de fundamentos el software de Atlas.ti nos indica cuales son los códigos con mayor fundamentación y de esa forma estructurar el proceso de aprendizaje en la concepción Qulla-Aymara.

Yatińa-Saber (41) precisamente es la categoría más fundamentada de toda la oralidad y es la más citada dentro de la oralidad recolectada, así como de las siguientes categorías. El saber es el subjeto de la presente investigación y como tal, es la esencia del del conocimiento Qulla-Aymara.

Amuyu-Pensamiento (39) acertadamente es la categoría que expresa un pensamiento desde etapas de desarrollo que define pensamientos $\mathrm{y}$ expresiones psicológicas del individuo.

Lurańa-Hacer (36) prácticamente es hecho mismo del ser QullaAymara a través de hermenéuticas establecidas por lógicas propias que son patrones de comportamientos Qulla-Aymara.

Yatiqaña-Aprender (32) sorprendentemente es una categoría esencialísima para poder alcanzar el éxito del proceso cognitivo Qulla-Aymara. La responsabilidad del ser vivo expresada en una actividad que da continuidad al colectivo social a través de procesos cognoscente.

Lup'ińa-razonamiento (21) racionalmente es un acto individual en busca de complementariedad, en repuesta los hechos de este espacio visible.

Sarnaqańa-Caminar (13) vivencialmente es vivir la vida en un constante proceso de aprendizaje por tiempos y espacios, por el cual, el ser recorre su vida en este universo.

Amtańa-Recuerdo (12) generacionalmente es lo que heredamos, genéticamente, culturalmente, intelectualmente, socialmente, económicamente, entre otras actividades del sarnaqaña.

Chuyma-Comportamiento (12) expresivamente es lo que damos a conocer desde el interior de nuestra vida, es la exteriorización del ser tal como quiere ser.

Jawq'ańa-Azotar (12) correctivamente si uno no se conecta con proceso de vida Qulla-Aymara necesita que sus conexiones neuronales sean cargadas de energía desde el exterior para poder conectar de forma correcta y encaminada en el sarnaqaña. 
ITodas las Redes Lup îĩa - Razonamiento, Chikuti - Garrote, Amtaña - Recuerdo-

ed Nodos Vinculos Diseño Presentación Especiales Ayuda

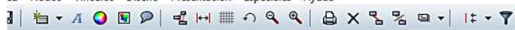

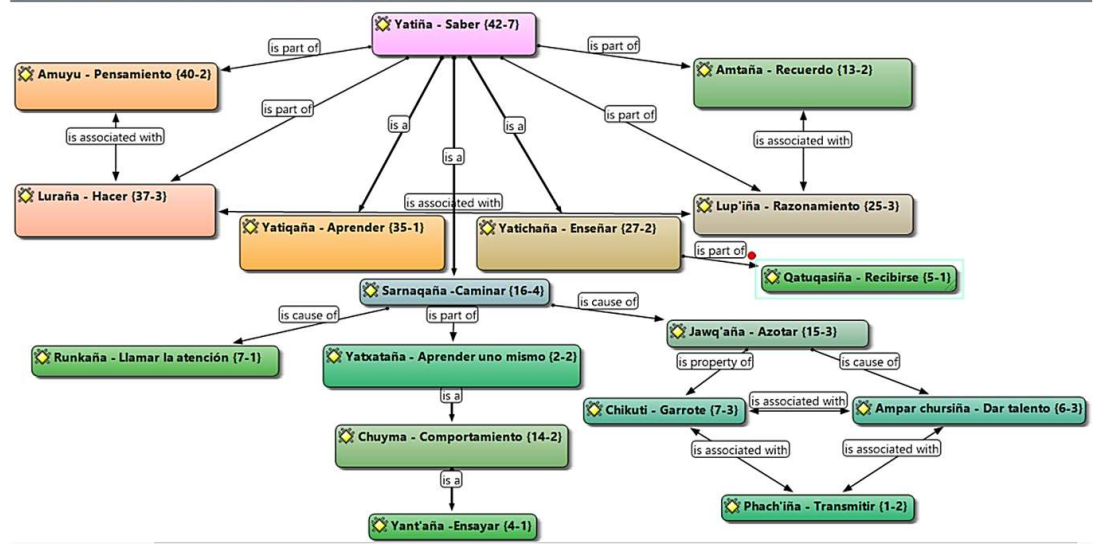

Las redes semánticas de yatińa nos muestran, cuatro redes semánticas que son parte de yatińa y estas actividades son: Amuyu-pensamiento, LurañaHacer, Lup'ińa-Razonamiento, Amtaña-Recuerdo. Las cuatro son redes semánticas están asociadas entre sí. El Yatiña-Saber es un Yatiqaña-Aprender y es un Yatichaña-Enseñar, y también es un Sarnaqaña-Caminar. El saber no se termina hasta el último día de tu vida, y si no caminas bien es a causa de Runkaña-Llamada de atención, y si en esa llamada de atención no te haces caso, es a causa o necesitas un Jawq'ańa-Azotar y es propiedad de un ChikutiGarrote, ¿por qué es una propiedad?, porque según los coinvestigadores a causa de ello podemos Ampar Churasiña-Dar talento. De esta forma la sociedad Qulla-Aymara está asociada con Phach'ińa-Transmitir. El Yatichańa-Enseńar es parte de Qatuqańa-Recibir es como un acto mezquino, es una dádiva por, lo tanto, uno se recibe lo encomendado, pero en muchas oportunidades no lo cumple o no se puede cumplir y solo es un cumplido nada más. En cambio, el Yatiqaña- Aprender es un deber como miembros de la sociedad Qulla-Aymara, si nadie piensa igual. no es igual que a su ayllu, automáticamente es otro. Si hubo algún sesgo al Yatiqaña-Aprender implica que no (Sabe) Yatiña-Saber.

El Modelo Educativo Qulla-Aymara extraída a partir de la teoría fundamentada demuestra que tienen cuatro pilares fundamentales, cuatro procesos que debemos seguir para alcanzar el Yatiña-Saber. Las cuatro secuencias son: 


\section{Amtaña-Recuerdo \\ 2. Amuyu-Pensamiento \\ 3. Luraña-Hacer \\ 4. Lup'ińa-Razonamiento}

Estudios anteriores ${ }^{6}$ lograron dar algunas pistas para poder reestructurar el modelo educativo Qulla Aymara.

El acto racional para una determinada actividad son precisamente el lup'i, el amuyu, el Lura. El orden de este proceso racional o de actividad cognitiva se desconoce posiblemente sea una de las más importantes para descubrir el proceso cognitivo de los aymaras hasta llegar al acto mismo de sus hechos. (17-1) (Cutipa Gomez, 2015)

A partir de la oralidad del Idioma Aymara de tres regiones (Chile, Perú y Bolivia) se ha podido aproximar a los conceptos que nos aproximan a poder dialogar con los hechos históricos registrados en cronistas, historiadores e intelectuales dedicados a la disciplina de la educación.

\section{A manera de conclusión}

Un modelo educativo es un molde social que perpetua la existencia de su ascendencia a partir de su descendencia. Un modelo educativo garantiza la continuidad del pensamiento y sentimiento de una Nación, Estado o País. La esencia de un modelo educativo es el alma de una cultura que pervive en el tiempo en una dinamicidad constante a partir del centro o de afuera hacia el centro.

En el discurso dentro la oralidad como el espíritu expresado a través de las palabras nos comunican el legado de la educación como molde para la continuidad y la construcción de la sociedad.

Los procesos del aprendizaje encontrados fueron organizados conforme a las secuencias del proceso cognitivo y conforme a la secuencia lógica se organizó y conceptualizó a partir de la oralidad de nueve informantes de distintos países a partir de los vecinos, códigos y co-ocurrentes.

\footnotetext{
${ }^{6}$ Tesis de Maestría. La concepción de proceso yati, en el mundo Qulla (Aymara) 2015
} 
1. Amtańa-Recuerdo, 'El recuerdo, haber, haber, hayque pensar pues, como nuestros padres caminaba. de ahí el niño como será, cómo lo hará realizará'

Entonces el proceso del yati 'saber' inicia con el amta el recuerdo de cómo eran nuestros padres, dentro de ello se encuentran el recuerdo genético, cultural, social, para ver que realizaron nuestros antecesores y que realizaran nuestros predecesores, en ningún momento se le obliga al miembro de una sociedad a ser parte tiene una ligazón, un parentesco y una obligación por ser parte de esta de la sociedad.

'¿A ver para hacer todo?, pensamos pues (amta), pensamos pues(lup'i)' Mä amtax intunsi taqinispay. V. Taqinixiy, ¿Un pensamiento (amta) entonces todos pueden ser? todos son pues'

"Amta aruxa. Nayax siristwa kunjams nayra pacha achachilanakas awichanakasa utxiritayna uk amtasina." 'la palabra amta, yo puedo decir como es el tiempo de nuestros abuelos y abuelas había habido eso recordando pues'

En síntesis, el amta es la genética legada por nuestros predecesores. La estructura genética Qulla-Aymara posee un legado de generación en generación, que tiene por herencia la forma de concebir la vida, coexistir la vida y dejar la vida. Toda actividad que realice debe consultar con el amta para poder pasar a la siguiente fase del proceso cognoscitivo.

2. Amuyu-Pensamiento, "tunka marankama, ukhax amuyasxiw" 'hasta los diez años, después ya tiene pensamiento (se da cuenta)'

El pensamiento tiene fases que debemos demarcar desde el vientre, nato, neonato, bebe, niño o nińa, jovenzuelo, hasta ahí está el amuyu presente, desde ahí es otro tipo de pensamiento. Lup'iñaya ukay Amuyaña 'razonamiento eso es pensar'

Primero debemos saber pensar y luego razonar el orden de estos dos procesos de pensar y razonar son dos componentes de un proceso de realizar (Luraña) de esta forma llegamos al yati (saber)

Amuyt'ańapax, ukat lup'itańapax wawanakamp amtasiñax, 'Deben pensar (amuyu colectivo) luego deben pensar (Lup'iña individual) con los hijos debemos pensar'.

aka jiwas amuyawi achachilanakasan laq'a achachilanakan jiwas laq'a achachilanakan amuyawix jan uñjat janiya amuyañjamakitixa. 
El Amuyu es un pensamiento colectivo, transmitido por el padreMadre, la sociedad y el entorno donde se convive. Por lo que, el amuyu es tanto abstracción y concreción subjetividad y objetividad.

3. Lup'ińa-Razonamiento, Kuntim awk tay lup'ki ukay lurixa. 'lo que piensa el padre la madre eso hace'.

“...amuyasxiw, tunka marata, es mi chica es mi chico, ukhamanak pinsxchijaya. ukhamarakis wawanak nuwi”

'ya piensa (amuyu) con diez años, chikujaw, chikajaw, así piensa (lup'i) a si son las wawas nové'

lup'ińaya ukay amuyañax 'razonamiento eso es pensar'

Primero debemos saber pensar y luego razonar el orden de estos dos procesos de pensar y razonar son dos componentes de un proceso de realizar (Luraña) de esta forma llegamos al yati (saber)

Lup'iski kuns lup'pachax, saranukuñ munpacha, jall ukhamaw lup'iski, pinsamiento. 'Esta razonando debe razonar pues, debe querer desviarse, de esta forma razona, el pensamiento es pues'

Ahora se entiende más el pensamiento como primer proceso es el pensamiento colectivo que no deja desviarnos, esta forma de razonamiento es de propiedad colectiva donde interviene el medio en el que uno vive. El lup'ińa como segundo momento del yati es el resultado de un entendimiento colectivo para poder razonar dentro la individualidad es un proceso donde cuestiona todo lo aprendido en la colectividada para luego pasar a una individualidad racionalizada pero no desligada de la realidad social, colectiva.

Awir kun lurañatakis awir J. Amyt'tanay, lup'tanay '¿A ver para hacer todo?, pensamos pues (amta), pensamos pues(lup'i)'

La relación entre el proceso ya está claro el primero es el amuyu luego es el lup'i, para luego demostrar con el Lura (hecho) como la concreción del pensamiento, el hacer el mostrar a través de los hechos de nuestro amta de nuestro recuerdo por un pensamiento con doble proceso.

V. L'up'isktan ukax amukï lup'isktan sapaki, jani taqpach akhamax isktan lup'isktan sapaki.

'Razonamos eso callado, razonamos solos, no todos así escucha, estamos razonando solos'

Confirmado el proceso del lup'i es un proceso individual. Un proceso reflexivo por el cual el amuyu no te deja solo debes tener pertenencia a una 
colectividad y de esta forma podrás realizar bajo el sustento del pensamiento colectivo.

Lup'isaw kuns apthapirakix jani kuns lup'kaspax janirakiw kuns lurkaspati 'Razonando puede recolectar, si no razona no puede hacer (luraña) nada'

Confirmado la secuencia o una forma de adquirir el conocimiento es amta, amuyu. lup'i y lura. El último como el hecho de un proceso final de un recuerdo, el pensamiento colectivo del medio que le rodea, el razonamiento individual que tiene sus componentes en el amuyu y poder realizar el lup'i y de esta forma la lura, el hecho mismo de un proceso yati.

“...pä tunk jila marani lup'iń yatxixa, he... dieceocho uka maranakan yasta pololiań ukaruw turasixa. Mas de 20 años ya sabe razonar diez y ocho desde esos ańos ya sabe pololear.

En síntesis, el proceso lup'i es el proceso racional que lo realiza de forma individual el joven que tiene completo el proceso de Amuyu, es decir que el pensamiento colectivo ya esta completo. El razonamiento es el proceso en el cual la experiencia, los conocimientos previos son sometidos a la prueba, frente a resoluciones y soluciones del proceso cognitivo que es el Lura.

4. Lurańa-Hacer, Uńaqasakipunirakiy luraskixa, observando (realizando todos los procesos) está haciendo.

“...ukay yatichañax wawanakarux akhamaw lurasiña sasa” 'eso debemos enseñar a los hijos, así debes hacerlo diciendo'

El enseñar haciendo se encuentra en el discurso de los aymaras, no existe aprendizaje sin hacer, sin el Luraña. Primero está el acto el hacer para que pueda aprender.

"Uñjapchix lurjapxakiyaps. Uñjasaki lurapxija”, observan pues y luego hacen nomas. Observando hacen.

inkarjatapiniya ukham ch'awata amuyumpiw ch'awata.

'está encargado siempre, así vas a hacer, pensando vas a ordenar'

Esto quiere decir que el pensamiento (Amuyu) es un pensamiento que se desarrolla en comunidad, observando y haciendo.

Lup'isaw kuns apthapirakix jani kuns lup'kaspax janirakiw kuns lurkaspati 'Razonando puede recolectar, si no razona no puede hacer (luraña) nada'

Confirmado la secuencia o una forma de adquirir el conocimiento es amta, amuyu. lup'i y lura. El último como el hecho de un proceso final de un 
recuerdo, el pensamiento colectivo del medio que le rodea, el razonamiento individual que tiene sus componentes en el amuyu y poder realizar el lup'i y de esta forma al Lura el hecho mismo de un proceso yati.

"Kuns jan wal lurapkta ukham jawk'añapunirinwa”. 'Que cosas no hemos hecho bien, de eso no azotaba siempre'

“...sum yatintańa sum luraña uk iwxirina” 'bie aprendido, bien hecho eso sabe encargarse'

"Ukanaka yatiqta uñaqasin kawkhimas jupha uk luririna, ukat uñaqasinay yatinxtaxa”. Esos he aprendido viendo como es, el así realizaba eso observando aprendemos.

El proceso cognoscitivo es la sumatoria de los cuatro procesos secuenciales y de esa forma llegamos al conocimiento, es decir al saber Yati o Yatińa Saber, verbo importantísimo en la actividad cognoscitiva del proceso Aprendizaje.

jisk'atpachasti jumax sum yatichanta, sum iwxäta kuna jan wali luripans k'utiqanta sum iwxäta suma pacha yatichata ukax jach'arux kuns suma lurani sasa ukhama arusisrina.

Desde pequeño tu bien debes enseñar, bien encargado, si algo sale mal, nuevamente bien se encarga tu mismo debes enseñar. Luego de grande cualquier cosa bien va hacer asi se encargaban.

Todo ese encargo les traemos ahora en este pequeño escrito para poder aproximar a un Modelo Educativo Qulla-Aymara.

Pay, pachiy, gracias, thanks.

\section{Referências}

ARNOLD, Y. D. Metodologías en las ciencias sociales en la Bolivia postcolonial: Reflexiones sobre el análisis de los datos en su contexto. En M. Yapu, INVESTIGACIONES CUALITATIVAS Y CUANTITATIVAS (págs. 3-106). La Paz: PIEB, 2010.

CUTIPA GOMEZ, N. La concepción del proceso yati en el Mundo Qulla (Aymara). La concepción del proceso yati en el Mundo Qulla (Aymara) Tesis CEPIES-UMSA. La Paz, Bolivia: UMSA, 2015.

CUTIPA GOMEZ, N. Tendencias Pedagógicas y la enseñanza de lenguas. La Paz: Talleres Gráficos Alarcón, 2013. 
LIMACHI, C. D. Estudio de la Toponimia Aymara y la interferencia del Castellano. Tesis de Grado . La Paz, Murillo, Bolivia: Universidad Mayor de San Andrés, 2011.

MORIN, E. La via para el futuro de la humanidad. Madrid: Paidos, 2011.

SOCIAL, M. d. Reporte comunal Colchane, Región de Tarapaca. Observatorio Social. Santigo : Ministerio de Desarrollo Social, 2014.

STRAUSS \& CORBIN, A. J. Bases de la Investigación Cualitativa. Técnicas y procedimientos para desarrollar la teoría fundamentada. Colombia: Universidad de Antioquia, 2002.

SUANNO, M. V. Didática e trabalho docente sob a ótica do pensammento complexo e da transdisciplinariedade. Didática e trabalho docente sob a ótica do pensammento complexo e da transdisciplinariedade. Brasília, DF: Programa de Pós-Graduacao Stricto Sensu em Educacao - UCB, 2015.

Recebido em: 20 ago. 2020

Aceito em: 17 set. 2020 